No $2002-16$

Décembre

Evolutions démographiques et marché du travail : des liens complexes parfois contradictoires

Loïc Cadiou Julien Genet

Jean-Louis Guérin 
Evolutions démographiques et marché du travail : des liens complexes parfois contradictoires

Loïc Cadiou

Julien Genet

J ean-Louis Guérin

$N^{\circ} 2002-16$

Décembre 


\section{TABLE DES MATIERES}

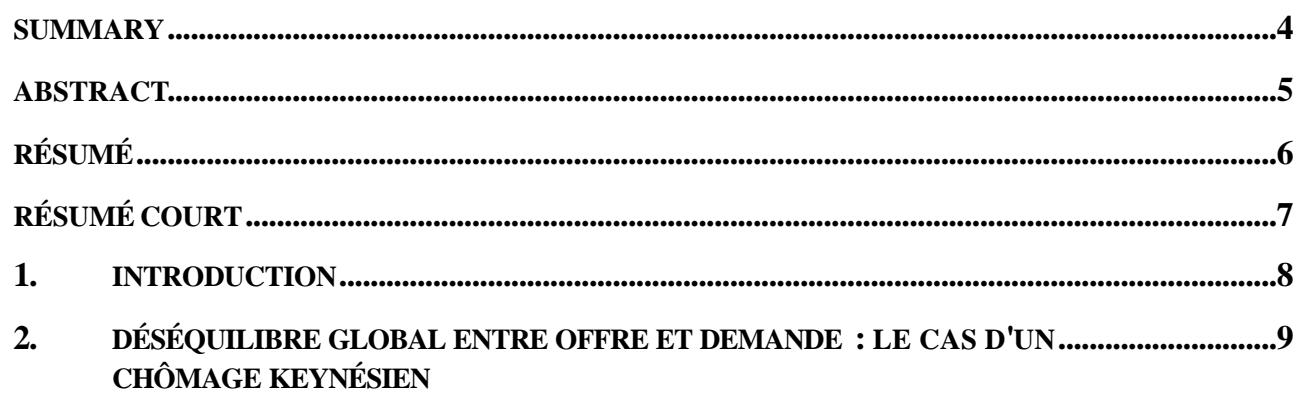

2.1. Le poids de la démographie est plutôt secondaire .............................................................12

2.2. l'effet du vieillissement sur l'épargne est peu avéré .........................................................12

3. DÉSÉQUILIBRE CLASSIQUE : LE ROLE DES MECANISMES DE FORMATION ................16 DES SALAIRES

3.1. Les modèles de chômage d'équilibre et les imperfections du marché ...........................17

3.2. Les effets d'hystérèse ou les mécanismes de persistance..................................................18

3.3. La courbe de Philipps et la rigidité salariale ...................................................................20

4. LA DEFORMATION DE LA STRUCTURE PAR ÂGE DE LA POPULATION ACTIVE.............21

4.1. Chômage des jeunes et des plus âgés : des logiques différentes ....................................22

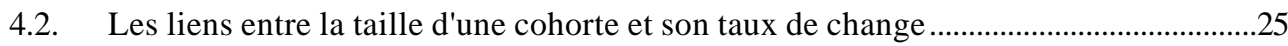

4.3. Vieillissement et rémunération à l'ancienneté

4.4. Salaires et taille d'une cohorte ........................................................................................2

4.5. Mobilité, qualification et productivité : quels liens avec l'âge .......................................28

5. CONCLUSION

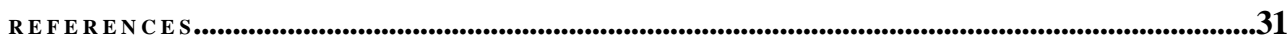

LISTE DES DOCUMENTS DE TRAVAIL REALISES PAR LE CEPII.........................................................35 


\section{DEMOGRAPHIC EVOLUTIONS AND LABOUR MARKET: COMPLEX AND CONFLICTING LINKS}

\section{SUMMARY}

This study focuses on the impacts of demographic evolutions on labour markets equilibrium. It is often tempting to draw a link between high growth in the active population and strong unemployment. This link is often cited to explain part of the difficulties on the employment front in Continental Europe. That leads to expect from the demographic break in trend in 2006 a new impetus towards full employment. That idea is nothing but the accounting Malthusian thematic, linking unemployment with too much labour supply.

Positions on this issue are still ambiguous. For some, that proposition is not convincing as empirical evidence does not support it. International comparisons do not show any significant relationship between the growth of the working age population and the level or the growth in unemployment rate. What is more, it is hard to see a logic behind the fact that a higher population would lead in the long run to higher unemployment.

But the persistent appeal of that approach still leads to expecting favorable outcomes from the 2006 break. That paradox needs to be addressed : this study tries to show the complexity of the links existing between demography and labour markets, under various assumptions on the equilibria on the latter. Whatever the framework chosen to look at those issues, it seems illusory to expect more than transitory effects from demographic evolutions. It looks like the labour market «adapts » to the growth rate of the working age population. Whatever that rate, unemployment seems always mainly brougth back to the level set by its other non-demographic determinants : economic cycle for Keynesians, labour costs and institutional rigidities for neo-classical practitioners.

At the same time however, evolutions in the age structure of the working age population could have non negligible consequences on the relative situation of some categories, but also on the prevailing rules on labour markets, such as seniority wage premia.

That analysis underlines the need to be cautious : if the forthcoming evolutions do constitute a real break, the issue of the needed policies to reach full employment remains to be addressed. 
Evolutions démographiques et marchés du travail : des liens complexes parfois contradictoires

\begin{abstract}
Evolutions forecasts on population, active population or their structure by age, are both a source for concern and optimism, raising fears on the financing of pensions systems or on the dynamism of the economy but also hopes of recovery on the unemployment front. The pensions issue has already been largely studied : population ageing raises the problem of balancing contributions rates, pensioners relative level of life and retirement age. But the other consequences of demographic evolutions are often left aside. This study focuses on the complex relations between demography and labour markets. While there are few doubts that the coming inversion in trend in the active population will constitute a major break, its impacts are uncertain : demography is only one of the determinants of macro-aggregates and its channels of transmission are many and sometimes contradictory. The only thing sure is that these evolutions won't be enough by themselves to ensure a return to full employment.
\end{abstract}

JEL Classification: $\mathrm{J} 10 ; \mathrm{J} 31 ; \mathrm{J} 41$

Key Words: $\quad$ Ageing, Demographic evolutions, Labour Markets 


\section{EVOLUTIONS DEMOGRAPHIQUES ET MARCHE DU TRAVAIL : DES LIENS COMPLEXES PARFOIS CONTRADICTOIRES}

\section{RÉSUMÉ}

Cette étude aborde la question de l'incidence des changements démographiques sur l'équilibre d'ensemble du marché du travail. On est souvent tenté de faire le lien entre croissance rapide de la population active et persistance d'un chômage élevé. Cette association reste assez souvent invoquée pour justifier une part de nos difficultés nationales en matière d'emploi. Et ceci conduit à attendre du retournement de 2006 un facteur d'embellie qui nous aiderait à reprendre la marche vers le plein emploi, interrompue depuis un an. Cette idée n'est rien d'autre que la thématique malthusienne et comptable qui lie chômage et trop plein de main d'œuvre.

Les positions vis-à-vis de cette thèse restent très ambiguës. Beaucoup sont convaincus qu'elle n'est pas pertinente. On dispose pour cela d'évidences empiriques : la comparaison internationale ne montre aucune relation significative entre intensité de la croissance de la population active et niveau ou croissance du taux de chômage. D'autre part, on ne voit pas les raisons logiques pour lesquelles une population plus élevée se traduirait, en régime permanent, par davantage de chômage.

D'un autre côté, la force de la logique malthusienne fait qu'on a du mal à se défaire totalement d'une vision favorable des effets du retournement de 2006. Ce paradoxe mérite qu'on y revienne : l'article s'attache à montrer la complexité des relations en jeu, en examinant les éclairages qu'y apportent différentes vision de l'équilibre du marché du travail. Il apparaît en fait que, quelle que soit la vision retenue, il est effectivement illusoire d'attendre plus que des effets transitoires du retournement démographique. Tout se passe comme si le marché du travail s' «habituait » au rythme de croissance de la population active. Que ce taux de croissance soit positif ou négatif, le taux de chômage apparaît plutôt attiré, comme par un aimant, par ses autres déterminants non démographiques : cycle économique pour les tenants des approches keynésiennes, coût du travail et rigidités institutionnelles pour les tenants d'une vision plus néo-classique.

Dans le même temps, cependant, les modifications de la structure par âge de la population active pourraient avoir des conséquences non négligeables sur les situations relatives de certaines catégories, mais aussi sur les règles en vigueur sur les marchés du travail telles que les politiques de rémunération à 1 'ancienneté.

Ce que nous montre l'analyse des effets potentiels de cette nouvelle donne pour le marché $\mathrm{du}$ travail, c'est qu'il serait sinon illusoire, du moins imprudent de compter sur une compensation mécanique de ce déséquilibre par la résorption spontanée du chômage. Dit en d'autres termes, le point de rupture de 2006 ouvre sans aucun doute une nouvel ère, mais cette perspective laisse entière la question des politiques requises pour reprendre la marche vers le plein emploi. 
Evolutions démographiques et marchés du travail : des liens complexes parfois contradictoires

\section{RÉSUMÉ COURT}

Les perspectives d'évolution de la population totale, de la population active et de leurs structures par âge sont alternativement sources d'inquiétude ou d'optimisme : craintes pour le financement des retraites, crainte d'effets négatifs sur le dynamisme de l'économie, ou, à l'inverse, espoir d'une forte amélioration des perspectives d'emploi. Parmi ces questions, celle de l'équilibre des retraites a été amplement balayée et est bien connue : le vieillissement de la population pose un incontestable problème d'arbitrage entre niveau des cotisations, pouvoir d'achat relatif des retraités, et âge de la retraite. Mais les autres conséquences des changements démographiques sont moins souvent explorées. Cet article se concentre sur la relation complexe entre évolutions démographiques et déséquilibres du marché du travail. Il fait peu de doutes que le retournement de tendance de la population active, au milieu de la décennie, va constituer une rupture majeure. Mais le sens des changements qui vont l'accompagner reste très incertain, à la fois parce que la démographie n'est qu'un déterminant parmi d'autres des comportements ou agrégats macroéconomiques, et parce que les canaux par lesquels agissent ces variables démographiques sont multiples et parfois contradictoires. La seule assurance qu' on puisse avoir est que le retournement de la population active ne saurait, à lui seul, garantir le retour spontané au plein emploi.

Classification JEL : J10; J31; J41

Mots-clefs : $\quad$ Vieillissement, Evolutions démographiques, Marchés du travail 


\title{
EVOLUTIONS DÉMOGRAPHIQUES ET MARCHÉ DU TRAVAIL : DES LIENS COMPLEXES PARFOIS CONTRADICTOIRES
}

\author{
Lö̈c Cadiou, Julien Genet, Jean-Louis Guérin
}

\section{INTRODUCTION}

L'évolution démographique à venir dans les économies industrialisées soulève de nombreuses interrogations. Le ralentissement de la population s'accompagnera-t-il d'une moindre croissance économique ? Le vieillissement de la population va-t-il conduire à l'émergence de conflits entre les générations, notamment sur la question des retraites et des systèmes d'assurance maladie ? De son côté, le ralentissement de la croissance de la population en âge de travailler contribuera-t-il à réduire le chômage ? Le vieillissement des travailleurs entraînera-t-il une modification de la formation des salaires selon la qualification et l'ancienneté ?

Plusieurs travaux récents ont abordé ces questions. On sait que la question des retraites a continué de donner lieu à de nombreuses publications, dont le premier rapport du Conseil d'Orientation des Retraites (COR, 2002). Les conséquences du retournement démographique sur les ressources en main d'œuvre et les façons d'y faire face sont également l'objet du rapport Quintreau (2002), et du rapport à paraître du groupe «Prospective des métiers et des qualifications » du Commissariat Général du Plan. La question des conséquences macroéconomiques générales des changements démographiques a été abordée, pour sa part, par Domingues Dos Santos (2001), Blanchet (2001). Un rapport plus récent du Conseil d'Analyse Economique a également abordé ce sujet (Aglietta, Blanchet et Héran, 2002).

Le présent article vient en prolongement de ces travaux avec un accent particulier sur les questions d'équilibre du marché du travail. Il le fait par une démarche en trois temps. Il pose d'abord la question de l'incidence des changements démographiques sur la demande : impact sur la demande globale et le partage épargne-consommation, en se situant donc plutôt dans une perspective de chômage keynésien. Cette première étape fait ressortir une première série d'indéterminations: les effets des changements démographiques sur la structure de la consommation sont en général plus faibles qu'on ne le pense, et leur impact sur le partage épargne-consommation et sur l'activité sont fortement tributaires des choix institutionnels qui seront faits pour adapter les systèmes de retraite à ces changements démographiques et des comportements d'investissement des entreprises.

CEPII, 9 rue Georges Pitard, 75740 Paris Cedex 15. Guerin@cepii.fr. Les auteurs tiennent à remercier Didier Blanchet pour les nombreuses améliorations effectuées sur une version préliminaire de l'article, ainsi que Agnès Bénassy-Quéré, Frédéric Lerais et Florence Legros pour les remarques apportées. La réalisation de cette étude a bénéficié du soutien financier de la DARES 
Evolutions démographiques et marchés du travail : des liens complexes parfois contradictoires

La seconde partie examine la même question dans un contexte de chômage classique, en mettant donc l'accent sur les mécanismes de formation des salaires. Selon la nature de ces mécanismes, l'impact du retournement démographique sur l'équilibre du marché du travail s'avère variable, mais les modèles accréditent, en général, plutôt l'idée d'une certaine indépendance à long terme entre taux de chômage et rythme de croissance démographique ou structure de la population.

Pour finir, la dernière partie approfondit l'analyse de l'effet des changements démographiques en prenant plus précisément en compte les différences de positions ou de comportements entre groupes d'âge: le retournement démographique affectera-t-il différemment actifs jeunes et actifs âgés ; est-il compatible avec le maintien des différences actuelles de niveaux de chômage ou de salaires par âge ; quelles seraient ses conséquences pour la fluidité du marché du travail?

\section{DÉSÉQUILIBRE GLOBAL ENTRE OFFRE ET DEMANDE: LE CAS D'UN CHÔMAGE KEYNÉSIEN}

Examiner l'impact des changements démographiques sur le niveau de chômage, c'est examiner l'impact relatif des changements démographiques sur l'offre et sur la demande de travail. On ne reviendra que brièvement sur le premier, qu'on supposera relativement mécanique. On sait bien sûr que le rapport peut-être relativement élastique entre évolution démographique de la population en âge de travailler et évolution de la population active, sous l'effet de variations des taux d'activité. Mais cette élasticité ne suffit pas à remettre durablement en cause la rupture de croissance prévue pour les années à venir, qui résulte essentiellement du basculement vers la retraite des générations du baby-boom. Ce sont au plus le moment ou l'ampleur de ce retournement qui peuvent être modifiés, mais il est certain que la longue période de croissance régulière de l'offre de travail à laquelle on a assisté depuis les années 1960 va progressivement prendre fin, et céder, au mieux, la place à une situation de stabilité ou de faible décroissance. Il n'aurait d'ailleurs pas été imaginable qu'il en aille autrement : une période de croissance rapide ne peut être qu'une parenthèse dans l'histoire d'une population, et il était normal que se referme progressivement la parenthèse de croissance correspondant à cette période du baby-boom.

Examiner l'incidence de ce même retournement démographique sur la demande de travail des entreprises est en revanche beaucoup plus complexe. La demande de travail des entreprises dépend en premier lieu du volume d'activité et de l'évolution du progrès technique. Elle est également fonction du coût réel du travail ou du coût du travail relativement au capital, selon la nature de l'équilibre macro-économique global. Dans un premier temps, ces effets de coût du travail sont néanmoins ici négligés pour privilégier l'analyse des effets des changements démographiques sur la demande de bien et services. 


\begin{abstract}
Encadré 1
Evolutions démographiques et structure de la demande de biens et services

La répartition de la consommation entre les différents biens et services dépend de l'âge. Les jeunes consomment relativement plus de biens d'équipement et les personnes âgées relativement plus de loisirs, de biens et services de santé, et de services de proximité. On peut dès lors s'attendre à une hausse de ces derniers types de dépenses dans la demande totale, ainsi qu'à une tertiarisation accrue de l'économie. Si les techniques de production sont différentes dans les secteurs concernés, notamment par leur intensité relative en travail et en capital, le changement de la structure de la consommation aura un impact sur la demande de travail des entreprises. Dans la mesure où la production de biens d'équipement est très intense en capital, la baisse de ce type de dépenses en faveur d'autres dépenses, dont la production est moins intense en capital, aura un effet favorable sur la demande de travail.
\end{abstract}

\title{
Les effets de l'âge et de la génération
}

De fait, l'étude des comportements de consommation conduit à distinguer trois groupes de consommation : les consommations dites « jeunes » (moins de 20 ans), celles de ménages d'âge moyen et celles des personnes âgées de plus de 65 ans (Darmon et al. 1991). Les consommations «jeunes »sont dans l'ordre décroissant les postes suivants : les transports, le loyer, les loisirs, l'habillement, les meubles et le tabac. Les consommations qui augmentent beaucoup avec l'âge concernent surtout le domaine de la santé.

Il faut cependant faire la part entre ce qui résulte d'un effet de génération et ce qui est effectivement dû au vieillissement. Par exemple, si l'on observe les dépenses des ménages, à un instant donné, on s'aperçoit que les dépenses de loisirs diminuent avec l'âge. Pourtant ce constat repose uniquement sur un effet de génération : parce qu'elles appartiennent sans doute à des générations qui sont nées avant l'essor des loisirs, les personnes âgées consomment moins de loisirs que les plus jeunes. Ainsi, si l'on suit les dépenses d'un ménage individuel au cours de sa vie, alors sa consommation de loisirs devient croissante avec l'âge.

En contrôlant ces effets de génération, l'effet du vieillissement sur la structure de la consommation des ménages français devient plus faible (Bodier 1999). L'effet propre à l'âge est faible mais significatif pour plusieurs postes de la consommation. Avec l'âge, les postes de consommation des ménages français qui diminuent le plus sont l'alimentation (7,3 en part de revenu pour les 60-64 ans par rapport au 40-44 ans), l'habillement (-6,7) ainsi que l'équipement du logement $(-3,3)$. Les postes qui augmentent le plus sont la santé $(+3,6)$, les loyers et charges $(+1,8)$ et les services domestiques $(+1)$. Cette déformation de la structure de la consommation s'accroît également avec l'âge. 
Ce constat se retrouve pour les ménages japonais. D'après une étude de The Sanwa Limited Bank (1994), la structure de la consommation des ménages de plus de 60 ans est peu différente de celle des 20-60 ans. Les résultats de cette étude s'expliquent sans doute en partie par le fait que les ménages âgés ne sont pas divisés en plusieurs groupes (les 60-64 ans, les 70-74 ans et les 80-84 ans) et que la structure de leur consommation est comparée à celle des ménages en général (et non par rapport à ceux des 40-44 ans). Le poste de l'éducation est comptabilisé dans la structure des dépenses des ménages. Ainsi, il représente, en 1989, 3,9\% des dépenses des ménages contre seulement 1,2\% de celles des ménages âgés. Inversement, le poids du poste des dépenses de santé des ménages âgés est supérieur de $35 \%$ au poids de ce poste chez leurs cadets.

\section{L'augmentation des dépenses de santé}

Plusieurs études consacrées au vieillissement de la population et aux dépenses des ménages américains insistent aussi sur l'augmentation des dépenses de santé. D'après Fuchs (1998), les dépenses de santé des américains de plus de 65 ans devraient représenter, sous l'hypothèse la moins forte, $10 \%$ du PIB des Etats unis en 2020. En extrapolant les tendances d'évolution des dépenses pour chaque classe d'âge, la consommation par tête relative à la santé atteindrait 25000 \$ (en dollars 95). D'après Hurd (1991) et Fuchs (1998) le revenu disponible pour la consommation d'autres biens et services devrait alors diminuer par rapport au niveau de 1995. Cette hausse des dépenses de santé est due pour un tiers à l'augmentation du nombre de personnes âgés et pour deux tiers à l'évolution de la consommation spécifique de chaque classe d'âge.

Ainsi, le vieillissement de la population semble avoir un impact limité sur la structure de la consommation. Il aurait un impact plus marqué pour la consommation de santé. Quelles en seraient les conséquences pour l'emploi global ? Il faut tenir compte du contenu de cette consommation en capital et en travail qualifié ou non qualifié. Or le domaine de la santé dans les dépenses des ménages est vaste. Il recouvre tout aussi bien l'achat de médicaments que la consommation de services de soins médicaux. Le secteur de la pharmacie est intense en travail qualifié (recherche et développement). Inversement, les services d'aide à domicile pour les personnes âgées dépendantes (exception faite des soins de santé à domicile) font appel à du travail peu qualifié. De même, le secteur de la santé recouvre à la fois des activités à forte intensité en capital (matériel technologique de pointe) et des activités à faible intensité en capital (service d'aide domestique et soins de santé à domicile). Cette disparité des activités de santé empêche de conclure à une forte importance des effets de composition en facteurs de production de cette évolution de la consommation. Cette évolution des dépenses de santé ne devrait cependant pas être sans conséquence : dans de nombreux pays, une bonne part de ces dépenses est assurée par l'état. Le vieillissement de la population devrait donc entraîner une modification de la composition des dépenses publiques ou un changement dans les systèmes de sécurité sociale, ce qui pourrait en retour affecter le taux d'épargne (on retrouve la discussion sur les retraites : les hausses de prélèvements sociaux pourraient affecter le marché du travail à la fois par leurs effets sur le coût du travail et sur l'épargne). 
L'impact indirect sur les marchés du travail de l'évolution de la structure de la consommation liée au vieillissement semble donc faible. Toutefois, la disparité des pyramides des âges actuelles entre professions pourrait créer des problèmes de renouvellement des effectifs dans certaines filières.

\subsection{Le poids de la démographie est plutôt secondaire}

En première approximation, la démographie a un impact simple sur la demande de biens et services. Si chaque individu a la même consommation alors on doit s'attendre à un ralentissement des dépenses totales de consommation proportionnel au ralentissement de la population totale. Si ce dernier est moins rapide que le ralentissement de la population active, ce qui sera le cas au cours des décennies à venir, alors cela signifie que la demande adressée à chaque actif est croissante. Sauf accélération de la productivité, ce différentiel de croissance entre demande de biens et offre de travail est favorable à la résorption du chômage. Ce n'est que lorsque le chômage bute sur son minimum incompressible que cette tension débouche sur un rationnement de la demande de biens. Tant que ce minimum n'est pas atteint, le retournement démographique est favorable au taux d'emploi.

La prise en compte du fait que la structure de la consommation dépend de l'âge modifie-telle ce premier raisonnement? Il est naturel de penser que les changements démographiques doivent se traduire à la fois par des réallocations sectorielles de la demande de travail, mais aussi, éventuellement, par une modification du niveau global de travail demandé, si le vieillissement pousse à la demande de services plus intenses en main d'œuvre. On constate toutefois (voir encadré 1) que les effets de la démographie sur la composition de la demande sont relativement faibles: les déterminants majeurs de l'évolution des structures de consommation à long terme sont à rechercher du côté des changements technologiques, qui ont à la fois des effets revenu (le progrès technique accroît le revenu global ce qui réduit la part des dépenses de première nécessité) et de prix relatifs (le progrès technique ne va pas au même rythme dans les différents secteurs et ceci modifie également la structure de la demande). Par rapport à ces tendances lourdes, le poids de la démographie est plutôt secondaire.

\subsection{L'effet du vieillissement sur l'épargne est peu avéré}

En revenant à une optique plus macro-économique, la question importante serait donc plutôt celle du partage global entre consommation et épargne, davantage que celle de la structure de la consommation. On s'attend en effet à ce que l'épargne varie avec l'âge. Une hypothèse classique, celle de cycle de vie, consiste notamment à supposer que si le jeune travailleur consomme en excès de son revenu (il a un faible taux d'épargne), le travailleur plus âgé est dans la situation inverse. Il épargne pour compenser sa faible épargne passée et accumuler un patrimoine financier en prévision de la baisse de revenu consécutive à l'arrêt de sa carrière professionnelle. Enfin, la période d'inactivité doit correspondre à une désaccumulation de cette richesse et donc à un taux d'épargne très faible.

Si une telle hypothèse était valide, la hausse de la proportion de retraités dans la population totale devrait donc avoir un impact négatif sur le taux d'épargne agrégé. Toujours selon la logique keynésienne, cet effet négatif sur l'épargne devrait être renforcer l'effet favorable 
Evolutions démographiques et marchés du travail : des liens complexes parfois contradictoires

du retournement démographique sur l'emploi et le chômage. Mais ce schéma est simpliste et doit être amendé sur de nombreux points.

D'une part, si la baisse de l'épargne peut-être favorable à l'activité économique et à l'emploi à court terme, elle est au mieux neutre à long terme : en abaissant l'accumulation elle abaisse à la fois les perspectives de production et d'emploi. Ensuite, l'hypothèse de cycle de correspond plutôt au cas d'un système de retraite par capitalisation, où chacun épargne pour sa propre retraite. Dans un système de retraite par répartition, les cotisations sociales jouent un rôle analogue à l'épargne puisqu'elles représentent un revenu différé ${ }^{1}$.

Encadré 2

\section{Impact du vieillissement de la population sur le taux d'épargne}

Parallèlement à l'évolution démographique, on observe une baisse du taux d'épargne dans les économies dites «vieillissantes ». Ainsi, il était de l'ordre de $20 \%$ au Japon et de $8 \%$ aux USA dans les années 70 et il est passé à $13 \%$ au Japon et à $5 \%$ aux USA dans les années 1990. Cette baisse du taux d'épargne peut en partie être expliquée par le vieillissement de la population en accord avec la théorie du cycle de vie présentée en troisième partie de cette étude.

\section{Baisse du taux d'épargne...}

$\mathrm{Au}$ niveau macro-économique, une plus grande proportion de personnes âgées dans la population déprime le taux d'épargne. En parallèle avec l'augmentation de la proportion de personnes âgées dans la population, on assiste à une diminution de celle des moins de 20 ans. Or cette diminution a une influence positive sur le taux d'épargne. Elle vient contrebalancer la pression à la baisse sur le taux d'épargne induite par l'augmentation de la proportion de personnes âgées. C'est pourquoi plusieurs études font intervenir le ratio de dépendance. Il s'agit du rapport entre le nombre de personnes âgées de moins de 20 ans et de plus de 65 ans sur le nombre des autres personnes, c'est-à-dire celles dont l'âge est compris entre 20 et $65^{2}$. Si ce ratio de dépendance augmente, alors l'effet devrait être négatif sur le taux d'épargne. C'est bien ce que trouvent Masson et al. (1996). La relation entre taux d'épargne et différentes variables dont le ratio de dépendance a été estimé à partir d'un panel de 21 pays industrialisés pour la période 1971-1993. L'effet du ratio de dépendance est négatif : une hausse de $10 \%$ de ce dit ratio provoque une baisse d'un peu plus de $1 \%$ du taux d'épargne. Ainsi, toujours d'après Masson et al. (1996), la hausse du sont ceux des rapports de consommation : la consommation d'un moins de 20 ans représente 0,7 fois celle des 20-65 ans; ce rapport est de 1,2 pour les plus de 65 ans.
} 
ratio de dépendance due au vieillissement de la population entraînera pour les trois prochaines décennies une baisse du taux d'épargne pour les pays développés .

\section{...alors que les ménages âgés désépargnent peu}

Au niveau microéconomique, le tableau dressé diffère : d'après des études réalisées sur des données concernant les dépenses des ménages (Weil 1994), la tendance à la désépargne des ménages «âgés ${ }^{4}$ » est faible voire nulle. En ce qui concerne les ménages français, cette tendance serait même inverse : à revenu et taille des ménages égaux, la consommation des personnes âgées est plus faible que celle de leurs cadets. Ceci peut s'expliquer par plusieurs effets : l'existence d'un système de retraites par répartition, un effet de génération et un effet d'âge (Bodier 1999). D'une part, les générations les plus anciennes ont toujours consommé moins que les générations leur succédant. D'autre part, avec l'âge, la consommation des personnes âgées diminue. Plusieurs arguments peuvent être avancés. Premier argument, avec l'âge, les besoins diminuent. Ensuite, la durée de vie étant inconnue, les individus constituent une épargne de précaution, pour ne pas vivre sans rien pendant les derniers moments de leur vie (la nature du système de retraites a donc un impact fort : la répartition en assurant une rente au travailleur retraité et à sa famille permet d'éliminer cet effet lié à l'incertitude sur la durée de vie). Enfin, cette diminution de la consommation peut refléter l'envie de laisser un héritage aux générations futures. L'hypothèse du cycle de vie selon laquelle les ménages «âgés » désépargnent est donc remise en cause.

Ainsi, à revenu et taille de ménage égaux, les 60-64 ans consomment 1.1 point supplémentaire de leur revenu que les 40-44 ans (voir tableau 3 en annexe). Cette part du revenu supplémentaire affectée à la consommation étant faible, on ne peut affirmer qu'il y ait une différence de comportement notable entre les ménages de 60-64 ans et ceux de 4044 ans. Par contre, à partir de 70 ans, le taux de consommation diminue de manière significative : 10,4 points du revenu des ménages de 70-74 ans par rapport à ceux de 40-44 ans ne sont plus affectés à la consommation. Cette baisse du taux de consommation est encore plus forte pour les ménages de 80-84 ans. Il n'y a donc pas ou très peu de différence entre les ménages français de moins de 60 ans et ceux dont l'âge est compris entre 60 et 70 ans ; des différences notables sur le taux de consommation interviennent uniquement à partir de 70 ans.

Loisy (1999) trouve que le taux d'épargne des ménages en France réaugmente à partir de 75 ans, même si il demeure inférieur à celui des tranches d'âge actives. Il est toutefois délicat de relier ces résultats à la théorie du cycle de vie : les ménages les plus aisés ont en général une espérance de vie plus longue mais aussi des comportements d'épargne différents des ménages moins aisés ; ceci pourrait expliquer l'apparente remontée des taux d'épargne passé un certain âge.

Pour les pays en développement, le ratio de dépendance évoluera dans le sens opposé. L'effet de l'évolution démographique sur l'épargne mondiale est donc ambigu.

4

On appelle par ménage « âgé » un ménage dont la personne de référence est âgée de plus de 60 ans. 


\section{Une contradiction seulement apparente}

Etudes micro-économiques et macro-économiques s'opposent donc quand à leurs prédictions de l'impact du vieillissement sur l'épargne. Un moyen de réconcilier ces deux phénomènes, apparemment contradictoires, a été apporté par Weil (1994). Les études sur données agrégées captent des effets inter-générations que les études micro-économiques ne peuvent saisir. Les ménages «jeunes » sachant qu'ils vont recevoir dans les prochaines années un héritage de la part de parents de leur famille augmentent leur niveau de consommation. Quant aux ménages âgés, ils désépargnent moins que prévu. Il en résulte macro-économiquement une baisse du taux d'épargne.

L'application de la méthode de Flaim (le calcul des effets directs de structure), déjà réalisée sur les taux de chômage et de participation, aux taux d'épargne mesurés par Loisy (1999) fournit des résultats de très faible ampleur (0,03 point d'épargne entre 1995 et 2020) : ce résultat provient de la similitude entre les taux d'épargne des ménages de moins de trente ans et de ceux de plus de 75 ans aux mouvements en volume opposés.

Si l'évolution démographique a donc un impact sur l'épargne, cet impact dépend fortement du système de retraite mis en place (Kohl et O'Brien 1998). Or l'organisation de ces systèmes de retraite est remise en cause par l'augmentation de la proportion de personnes de plus de 60 ans. L'impact résultant sur l'épargne doit donc tenir compte des changements institutionnels dans les systèmes de retraite.

Si les actifs jugent que leurs cotisations sont suffisantes pour leur fournir un revenu satisfaisant pendant leur période d'inactivité, ils ne modifient pas leur comportement d'épargne une fois inactifs, et il n'y a pas à s'attendre à un effet important du vieillissement sur l'épargne, ni positif ni négatif.

D'ailleurs, même dans les pays où le système par capitalisation domine, cette vision simple d'une baisse du taux d'épargne par tranche d'âge est loin de faire l'objet d'un consensus. Les études empiriques sont relativement contradictoires (voir encadré 2). Il semble plus raisonnable de considérer que le taux d'épargne des jeunes inactifs est nul, que celui des actifs jeunes est effectivement faible, que celui des actifs âgés est élevé, mais que pour des raisons de transfert intergénérationnels privés (héritage) ou d'incertitude sur la durée de vie, les inactifs âgés ne réduisent guère leur effort d'épargne. Au total, la hausse de la proportion de retraités dans la population totale peut ne pas avoir l'effet structurel attendu de baisse du taux d'épargne national. On reviendrait à l'idée de départ selon laquelle la demande évoluerait, en gros, de manière proportionnelle à la population totale.

Enfin, même si l'on croit à un modèle de cycle de vie pur, les effets du vieillissement sur l'épargne doivent aussi être différenciés selon l'horizon auquel on se place. Supposons que, en réponse au vieillissement, le taux de remplacement des retraites baisse progressivement. Dans ce cas il faut distinguer deux phases. L'une ou les actifs augmentent leur épargne en prévision de cette baisse sans qu'il y ait augmentation simultanée de la désépargne des retraités, qui continuent à bénéficier de leurs droits acquis. Dans ce cas, le premier effet est une hausse plutôt qu'une baisse de l'épargne. Ce n'est que dans un deuxième temps que cet épargne additionnelle se transforme en désépargne et que le taux d'épargne peut donc 
s'abaisser, mais il n'est pas sûr qu'il revienne à un niveau inférieur à celui qui était le sien avant que ne démarre le processus de vieillissement. Prenons par exemple le cas simple d'un vieillissement par allongement de la durée de vie. La désépargne plus forte des retraités plus nombreux n'est que la contrepartie d'une épargne supplémentaire d'actifs qui anticipent une durée de retraite plus longue : les deux phénomènes s'équilibrent et le vieillissement est parfaitement neutre pour l'épargne à long terme.

Au total, l'idée d'un effet négatif du vieillissement sur l'épargne est contestable et n'est au mieux valable que pour le long terme. Au cours de la phase transitoire, on peut tout aussi bien assister à une croissance plutôt qu'à une décroissance de l'épargne, et, toujours dans un cadre strictement keynésien, l'incidence sur l'activité et l'emploi serait inverse de ce que donnait le raisonnement initial. Cet effet dépressif sur l'activité sera d'autant plus marqué si, face à cette hausse de l'épargne, la demande d'investissement est faible. Par exemple, les entreprises peuvent anticiper que le ralentissement démographique va freiner leurs débouchés, et réduire donc leur investissement en conséquence. Dans ce cas, l'impact du choc démographique peut aller jusqu'à une contraction de l'activité et une détérioration plutôt qu'une amélioration de l'emploi. Il est certes possible de corriger le déséquilibre épargne-investissement qui est à l'origine de cette contraction de l'activité par l'exportation de l'épargne. C'est le scénario dans lequel l'épargne retraite des actifs irait s'investir à l'étranger: ce scénario reste néanmoins défavorable à l'activité intérieure. Il n'y a que dans le cas où ces investissements à l'étranger financeraient un montant exactement équivalent d'exportation vers les pays concernés que le déséquilibre ex ante entre épargne et investissement pourrait être résolu sans aucune réduction de l'activité, ce qui est une hypothèse un peu extrême.

\section{DÉSÉQUILIBRE CLASSIQUE : LE RÔLE DES MÉCANISMES DE FORMATION DES SALAIRES}

Une approche keynésienne élémentaire qui ne raisonne qu'en termes de quantités ne garantit donc pas qu'un retournement démographique permette l'amélioration du taux d'emploi. Qu'en est-il lorsqu'on fait intervenir les mécanismes d'ajustement des prix et des salaires et/ou l'hypothèse d'un chômage classique lié à des niveaux de salaires trop élevés ?

Le raisonnement de base est le suivant. On peut repartir de l'idée que la baisse de l'offre de travail se traduit ex ante par une baisse équivalente du nombre de chômeurs. Mais cette baisse entraînera une hausse du salaire réel, qui, en retour, réduit la demande de travail des entreprises. Ex post, la baisse du chômage devra donc être inférieure à celle de l'offre de travail. Cet effet sur l'emploi est d'autant plus prononcé que les salaires réagissent fortement à la hausse. A la limite, lorsque l'ajustement des salaires est immédiat, la demande de travail des entreprises diminue comme l'offre, et le chômage est inchangé.

Ainsi, la nature et l'importance des ajustements salariaux sont au cœur de l'analyse du taux de chômage à moyen et long terme. Elles continuent de faire l'objet de nombreux débats parmi les économistes. Sans trancher entre les différentes approches de ces questions, ce qui sort évidemment de l'ambition de cet article, on peut se contenter plus simplement de confronter les messages auxquels elle conduisent en matière d'incidence des changements démographiques. On distinguera trois grandes approches : celle des modèles dits de 
Evolutions démographiques et marchés du travail : des liens complexes parfois contradictoires

chômage d'équilibre, dans lesquelles le taux de chômage est totalement déterminé par le contexte institutionnel, celle des modèles à effet d'hystérèse, où le niveau courant du chômage résulte en partie des évolutions économiques passées, et enfin l'approche, plus traditionnelle mais purement empirique, dérivée de l'équation de Philips.

Tout naturellement, il faudra ajouter à ces mécanismes les possibles répercussions d'une évolution des systèmes de retraite : ainsi, si les taux de cotisation, des salariés comme des employeurs, sont revus à la hausse comme part d'une solution destinée à préserver le système par répartition, alors un effet «coin fiscal» s'ajoutera aux effets décrits précédemment ${ }^{5}$.

\subsection{Les modèles de chômage d'équilibre et les imperfections de marché}

Sous l'hypothèse de parfaite flexibilité des prix et des salaires, le chômage ne peut résulter que des imperfections du marché du travail (asymétrie d'information entre le travailleur et l'entreprise, contraintes de licenciement, négociation collective). Ces imperfections génèrent une rente, captée par les salariés, de sorte que le salaire se fixe au-dessus du salaire d'équilibre concurrentiel ${ }^{6}$. On parle alors de "chômage d'équilibre " (Layard et al. 1991). Le chômage peut être qualifié d'involontaire au sens où les demandeurs d'emploi sont prêts à accepter un emploi pour un salaire équivalent au salaire courant, voire inférieur, mais n'en trouvent pas.

Le chômage peut également résulter d'une rigidité réelle des salaires dont l'origine ne serait pas limitée aux imperfections du marché du travail. Mais comme précédemment, le chômage joue le rôle d'incitation à accepter un certain niveau de salaire réel. Le chômage résulte alors de divers chocs ayant affecté chaque économie : hausse des taux d'intérêt réels, du progrès technique, ralentissement de la croissance de la productivité du travail (en Europe), augmentation des allocations chômage, des coûts de licenciements, des taxes sur les salaires, du salaire minimum, chocs pétroliers (Phelps, 1994 ; Phelps et Zoega, 1998).

Ces types de fonctionnement du marché du travail auxquels sont associés la notion de chômage structurel/d'équilibre peuvent être représentés simplement au niveau macroéconomique. Le salaire réel accepté par les travailleurs s'accompagne d'un certain chômage, puisqu'il est supérieur au niveau équilibrant l'offre et la demande notionnelles de travail. Ici le niveau des salaires réels est lié au niveau du taux de chômage.

Dans ces modèles, la baisse de l'offre de travail se traduit par une baisse ex ante du chômage. Toutefois, les travailleurs n'acceptent alors d'offrir leur travail que pour un

\footnotetext{
5 Château et al. (2002) fournissent un descriptif de ces effets dans le cadre d'un modèle d'appariement appliqué à la France. 6

${ }^{6}$ Dans le modèle de salaire d'efficience, ce sont les entreprises qui fixent volontairement le salaire à un niveau élevé afin d'attirer les travailleurs les plus efficaces et de les inciter à maintenir leur effort une fois embauchés Au salaire réel d'équilibre, une proportion de la force de travail ne trouve pas d'emploi. Les entreprises refusent de réduire le salaire réel en deçà d'un niveau auquel la productivité d'un travailleur supplémentaire, corrigée de son incitation salariale à l'effort, devient inférieure à son coût. Le chômage constitue en retour une incitation pour les personnes employées à poursuivre leur effort. Il est également de nature involontaire.
} 
salaire réel plus élevé. L'impact ex post sur le chômage dépend des caractéristiques de la demande de travail des entreprises et du salaire demandé par les travailleurs. Dans les modèles de chômage d'équilibre, le salaire accepté par les travailleurs résulte d'un processus de négociation entre les entreprises, qui veulent maximiser leur profit, et les travailleurs ou leurs représentants, qui désirent maximiser une combinaison des salaires et, éventuellement, de l'emploi. Ainsi, le niveau du salaire réel se déplace en partie comme la demande de travail des entreprises. L'effet net d'une baisse de l'offre de travail sur le chômage est ambigu ${ }^{8}$. Il est toutefois a priori faible, puisque les principaux déterminants de ce type de chômage se trouvent dans les imperfections des différents marchés. Seules des réformes institutionnelles ou réglementaires, ou encore l'amélioration des mécanismes d'incitation peuvent influencer le niveau du chômage. A ce niveau de chômage, l'économie a atteint une forme de plein emploi.

Les estimations économétriques d'équation de salaires en niveau dans les pays de l'OCDE (voir en particulier Tyrvainen, 1995) indiquent que l'indexation des salaires réels sur la productivité moyenne du travail est très imparfaite. Dans ce cas, l'effet d'une baisse de l'offre de travail est susceptible d'entraîner une certaine diminution du chômage structurel. Mais, le trait marquant de ces estimations est la lenteur de l'ajustement du niveau des salaires face à une modification du chômage. En d'autres termes, la relation entre le niveau des salaires et le niveau du chômage ne prendrait effet qu'à partir d'un certain horizon. Elle laisserait entre temps la place à des dynamiques de moyen terme de nature différente. On présente dans la suite deux types d'ajustements dynamiques. Le premier emprunte aux imperfections de marché qui viennent d'être présentées. Le second repose sur une représentation essentiellement empirique de la formation des salaires.

\subsection{Les effets d'hystérèse ou les mécanismes de persistance}

Le maintien d'un chômage élevé en Europe depuis le milieu des années 80 a donné lieu à une autre série d'explications dont le point commun est de se focaliser sur les mécanismes de persistance. C'est le cas notamment de la théorie insider-outsider. Celle-ci s'appuie sur la distinction que créent les imperfections du marché du travail entre les travailleurs en place (les insiders) et les autres (les outsiders). Ces imperfections (asymétrie d'information, coût d'embauche et de licenciement, contraintes légales sur les licenciements) sont telles que les insiders ne peuvent être remplacés par des outsiders sans coût pour l'entreprise. Ainsi, les premiers réussissent à capter une partie des profits réalisés par l'entreprise. En période d'expansion, les insiders obtiennent des hausses de salaire (Blanchard et Summers, 1986, Lindbeck et Snower, 1988, Kidd et Oswald, 1987) supérieurs aux gains de productivité du travail. L'embauche d'oustsiders peut avoir lieu, mais ces derniers viennent gonfler les rangs des insiders de la période suivante et ainsi renforcer le pouvoir des salariés en place lors de la détermination des nouveaux salaires. Ainsi les phases d'expansion sont

\footnotetext{
7 Le degré d'indexation du salaire réel sur la productivité moyenne ou marginale du travail détermine l'ampleur de ces mouvements.

8

Si le facteur travail est à rendements décroissants, la productivité moyenne est supérieure à la productivité marginale, ce qui peut compenser la plus faible indexation des salaires réels négociés sur la productivité.
} 
Evolutions démographiques et marchés du travail : des liens complexes parfois contradictoires

surtout caractérisées par une hausse des rémunérations et peu de créations d'emplois. Le chômage baisse peu en période de croissance. En période de récession en revanche, les entreprises ajustent à la baisse l'emploi et donc le nombre d'insiders. Dès que ces derniers anticipent la fin de la récession, ils captent les gains de productivité sous forme de salaire, de sorte qu'à l'issue d'un cycle économique le niveau d'emploi aura baissé.

Une conséquence de cet effet d'hystérèse ${ }^{9}$ est que le niveau du chômage courant d'un pays résulte de son histoire économique. A court terme, le chômage ne peut baisser trop rapidement. A moyen terme toutefois, l'évolution du chômage dépend de la vitesse de convergence de l'économie vers son niveau de plein emploi (ou de chômage d'équilibre).

La distinction entre les travailleurs ayant un emploi et les autres n'est pas la seule explication possible de la persistance du chômage à moyen terme. Le phénomène de démotivation et de déqualification des chômeurs est également susceptible de produire de l'hystérèse. Lorsqu'un travailleur reste trop longtemps sans emploi, il perd progressivement le contact avec le marché du travail. D'une part, ses aptitudes sont moins reconnues par les employeurs. D'autre part, ses aptitudes sont de moins en moins adaptées aux besoins nouveaux des entreprises. Le temps passé au chômage est également un signal pour les employeurs éventuels.

Dans ce type de modèle des chocs transitoires ont des effets durables car le déséquilibre initial induit une modification du comportement des agents (insiders, chômeurs de longue durée) qui pérennise le déséquilibre. Mais ces effets ne sont pas symétriques (d'où l'hystérèse). En effet un choc opposé au choc de départ ne permet pas de revenir à la situation initiale.

En ce qui concerne l'effet d'une baisse de l'offre de travail, les modèles précédents conduisent ex ante à une baisse du chômage. Le modèle insider-outsider correspond à un arbitrage salaires/embauches favorable aux salaires. La prise en compte d'un moindre risque de chômage peut conduire à une hausse des salaires au détriment de l'emploi. L'effet favorable sur le chômage de la baisse de l'offre de travail serait atténué, voire annulé, par l'effet négatif sur l'emploi. Dans le modèle avec processus de déqualification/démotivation progressive des chômeurs, la baisse de l'offre diminue le nombre de chômeurs nouveaux les périodes suivantes, de sorte que la durée moyenne de chômage baisse, ce qui cette fois est favorable à l'emploi. En plus de l'effet direct sur le niveau du chômage, toutes choses égales par ailleurs, le ralentissement de l'offre de travail atténuerait dans ce cas les phénomènes de démotivation/déqualification.

Au total, la dynamique du chômage face au ralentissement de l'offre de travail dépend des mécanismes de persistance retenus. D'un côté, le nombre d'outsiders diminue ce qui renforce la capacité des insiders à obtenir des hausses de rémunérations. Le ralentissement de l'offre de travail n'a alors aucun effet sur le chômage qui se maintient à son niveau courant. D'un autre côté, la durée moyenne du chômage baisse ce qui améliore la situation des outsiders dans leur recherche d'un emploi (qualification encore adaptée, motivation

\footnotetext{
9 L'hystérésis correspond ici au fait qu'à l'issue de deux chocs successifs et opposés, l'économie ne retrouve pas son état initial.
} 
intacte et prise en considération par les employeurs potentiels). Ceci accroît la concurrence entre insiders et outsiders. L'évolution du chômage dépend finalement de l'importance relative des dynamiques précédentes. On peut toutefois retenir qu'en présence d'effets d'hystérèse, le ralentissement démographique ne peut s'accompagner, au mieux, que d'une baisse progressive du chômage.

\subsection{La courbe de Phillips et la rigidité salariale}

Comme cela a déjà été évoqué plus haut, les estimations d'équations de salaires en niveau sont caractérisées par un ajustement lent aux tensions sur le marché du travail. La courbe de Phillips constitue une représentation alternative de la rigidité salariale, reliant cette fois le taux de croissance des salaires au niveau du chômage. Il s'agit d'une modélisation plus ad hoc des imperfections du marché du travail et du mode de formation des salaires ${ }^{10}$, mais également plus satisfaisante sur le plan empirique.

La principale différence avec une relation entre le niveau des salaires et le niveau du chômage (modèle WS) concerne le chômage de moyen terme. A court terme, les deux formulations peuvent rendre compte d'un chômage de déséquilibre (de nature keynésienne ou classique). A moyen terme, ce type de chômage disparaît avec la formulation en niveau, alors qu'elle peut persister avec la formulation en taux de croissance ${ }^{11}$ (courbe de Phillips). Dans ce dernier cas, les salaires dépendent en effet de l'histoire économique du pays considéré. Contrairement au modèle WS, ils ne dépendent pas seulement des conditions de marché courantes.

Compte tenu de la rigidité salariale dont rend compte la courbe de Phillips, les salaires n'équilibrent pas le marché du travail. Un rationnement s'opère sur ce marché. Selon l'importance relative de la rigidité des prix et des salaires, le chômage sera de nature classique ou keynésienne. Dans les deux cas, une baisse de l'offre de travail réduit le rationnement sur le marché du travail, donc le niveau du chômage.

En conclusion, les évolutions démographiques en volume ne devraient guère avoir d'impact sur le niveau de chômage de long terme. L'effet du ralentissement de la population active sur le chômage de moyen terme dépend de l'importance de la rigidité des salaires et des prix. Lorsque les prix et les salaires sont assez flexibles, le chômage de moyen terme est essentiellement influencé par les régulations du marché du travail. Les évolutions démographiques ne peuvent alors jouer qu'un rôle indirect par des arguments empruntés à l'économie politique ; elles pourraient par exemple conduire à des réformes du marché du travail ou des modifications importantes des rapports de forces entre les acteurs économiques.

10 La courbe de Phillips peut être vue comme une relation macro-économique rendant compte grossièrement des rapports de forces entre salariés et employeurs en fonction de l'état du marché du travail. Elle est également proche d'une spécification d'un modèle de négociation des salaires dans lequel le salaire de réservation est égal au salaire courant précédent (Cahuc et Zylberberg, 1999). 11

Selon la spécification retenue, le NAIRU peut dépendre ou non de l'offre de travail (et donc de l'évolution démographique). 
Evolutions démographiques et marchés du travail : des liens complexes parfois contradictoires

Lorsque les prix et les salaires sont rigides, le ralentissement de la population active peut au contraire s'accompagner d'une baisse sensible du chômage à moyen terme. Les prix et les salaires ne répondant que très imparfaitement aux déséquilibres sur les marchés, la baisse de l'offre de travail réduit le rationnement sur le marché du travail.

Enfin, la prise en compte d'effets d'hystérèse implique une rigidité à la baisse du chômage. Celle-ci peut être totale ou partielle. Dans ce dernier cas, le rythme auquel le chômage diminue en réponse au ralentissement de la population active est un facteur important de la baisse finale du taux de chômage à moyen terme.

Ces analyses, cependant, ne sont pas rentrées dans le détail de la composition par âge de la population active, or l'examen des données de chômage et de salaires montre que ceux-ci sont fortement différenciés selon l'âge.

\section{LA DÉFORMATION DE LA STRUCTURE PAR ÂGE DE LA POPUlation ACTIVE}

La prise en compte de ces éléments de composition suggère tout d'abord une autre forme d'incidence des changements démographiques sur le chômage moyen. Par exemple, si le chômage est décroissant avec l'âge, alors le vieillissement de la population active devrait se traduire par une baisse du taux de chômage moyen. Il est facile de montrer que cet effet de composition est en fait limité (encadré 3): les raisons sont les mêmes que celles qui expliquaient la faible sensibilité des structures de consommation par rapport à la structure par âge de la population. Même si le lien entre âge et exposition au risque de chômage existe, les déformation prévisibles de la structure par âge de la population sont d'une amplitude trop faible pour que ceci se traduise par un changement important du taux de chômage moyen.

En revanche, ces différentiels de chômage invitent à souligner que les causes de chômage ne sont pas les mêmes ou ne jouent pas avec la même ampleur pour tous les groupes d'âge, et donc à examiner si les chocs démographiques ne seraient pas susceptibles d'affecter différemment l'accès à l'emploi ou le maintien de l'emploi des différentes catégories de population. L'examen de ces effets, et de leur transmission ou non entre catégories, nécessite aussi de se poser la question de la substituabilité des différentes cohortes. Or, malgré leurs différences de caractéristiques ou de comportement, les différentes classes d'âge sont quand même partiellement substituables entre elles ${ }^{12}$. La prise en compte d'une telle substituabilité peut modifier les impacts attendus : la taille relative d'une cohorte affectera, si elle a un effet, non seulement la situation économique à l'intérieur de cette cohorte, mais aussi à terme celle des autres cohortes.

Enfin, au delà des salaires et du taux de chômage, les salariés diffèrent entre eux par des caractéristiques susceptibles de modifier l'équilibre du marché du travail telles que la

12 Pour l'OCDE (1998), une variation de la structure de la pyramide des âges n'aurait eu aucun impact ni sur le chômage moyen entre 1970 et 1996, ni sur la variation du chômage entre ces deux dates, ce qui sous tendrait l'existence d'une parfaite substituabilité sur longue période entre les différentes classes d'âge. 
mobilité ou la productivité (ce dernier impact potentiellement d'autant plus important qu'il renvoie à la question du lien entre croissance économique et croissance démographique).

\subsection{Chômage des jeunes et des plus âgés : des logiques différentes}

Le chômage des actifs jeunes provient de plusieurs sources : il existe un effet file d'attente qui s'explique par des mécanismes insiders/outsiders ; de même, les jeunes sont plus exposés aux chocs (« last in, first out») ; enfin et surtout, les actifs jeunes subiraient plus que leurs aînés, de par leur inexpérience, les effets négatifs de coûts du travail trop élevés. Brown et al. (1982) affirmaient ainsi qu'une hausse de $10 \%$ du salaire minimum aux USA se traduisait par une diminution de l'emploi des adolescents d'environ $2 \%$ et n'avait aucun effet significatif sur le marché du travail des salariés plus âgés ${ }^{13}$. Dolado et al. (1996) confirment ce résultat pour l'Europe. Enfin, l'OCDE (1998) aboutit aux mêmes conclusions : le salaire minimum aurait un impact macroéconomique négligeable; en revanche, il aurait un impact négatif significatif sur l'emploi des jeunes, en particulier des non-qualifiés.

Le chômage des actifs âgés répond à une logique différente. Les actifs âgés au chômage ne retrouvent que rarement un emploi car leur mobilité et leur flexibilité sont moindres et car leurs demandes salariales sont souvent trop élevées : l'ancienneté est valorisée en interne, pas sur le marché externe, et, pour la firme, ils ne resteront pas suffisamment longtemps pour justifier les coûts d'adaptation. En conséquence de quoi, nombre d'entre eux choisissent tout simplement de quitter la population active. Cette décision ne transparaît pas dans les taux de chômage mais dans les taux de participation. Elle est déterminée par le montant des retraites personnelles et les problèmes de santé (Clark et Spengler 1980), par le montant des allocations vieillesse, par l'existence de mécanismes de départ en pré-retraite et enfin par le chômage général qui décourage les actifs âgés de chercher un travail (Diamond et Hausman 1984). De plus, l'inadéquation entre offre et demande de travail est plus marquée pour les âgés (non-maîtrise des nouveaux outils, etc.). Enfin, il faut noter que le déroulement des fins de carrière s'est profondément modifié : en France, 15\% des retraités ayant actuellement entre 60 et 64 ans ont été en préretraite, dont les trois-quarts pour des durées supérieures à trois ans ; de plus, en 1992, un licenciement économique sur trois a touché un salarié de plus de 50 ans (Marchand et Salzberg, 1996).

\footnotetext{
13

Les effets du salaire minimum sur l'emploi et le chômage ne font cependant toujours pas l'objet d'un consensus.
} 
Evolutions démographiques et marchés du travail : des liens complexes parfois contradictoires

Encadré 3

\section{Structure par âge de la population active et taux de chômage moyen}

Le chômage touche principalement les jeunes (surtout les non-diplômés) dans les sociétés industrialisées. Ces différences de taux de chômage peuvent être notables : à titre d'exemple, aux USA en 1979, ce taux était de $11,8 \%$ pour les moins de 25 ans et de 3,9\% pour les plus de 25 ans. Ce constat peut nous permettre de calculer en équilibre partiel un premier impact de l'évolution démographique sur le chômage agrégé qui ignore les causes derrière ces différences dans les taux de chômage.

\section{Les effets de la composition par groupe d'âge de la population...}

Le taux de chômage global étant égal à la somme des taux de chômage des différentes cohortes pondérés par leurs poids, il suffit de mesurer l'impact de l'évolution du poids des différentes cohortes pour avoir une première approximation, les taux de chômage des différentes cohortes étant ici supposés invariant, des effets d'un vieillissement de la population (la même méthode peut être appliquée aux taux de participation qui diffèrent eux aussi entre cohortes).

Flaim (1990) applique cette méthode aux USA pour tenter de mesurer l'impact de l'arrivée sur le marché du travail de la génération du baby boom, puis celui de son vieillissement. Entre 1959 et 1979, la proportion de jeunes actifs de moins de 25 ans passe de 16 à $24 \%$. Le chômage des jeunes étant supérieur à celui du reste de la population (11\% contre $4,4 \%$ en 1959), l'effet démographique direct est donc un accroissement de 0,5 point de chômage. Son effet est partiellement compensé par la baisse du chômage chez les plus de 25 ans. Le chômage n'augmente donc que de 0,3 point (de 5,5\% à 5,8\%). Dans les années 80 , l'évolution démographique conduit à une hausse du poids relatif de la catégorie des 25-45 ans ; le taux de chômage de cette classe d'âge étant inférieur au taux moyen, l'évolution démographique participe ici à la décrue du chômage. Elle suffit même à expliquer la décrue observée de 0,5 point du chômage entre 1979 et 1989 de 5,8\% à 5,3\%. Elle n'explique par contre que $20 \%$ des évolutions observées du taux de participation. Les prévisions de Flaim pour les années 90 indiquaient une décrue du chômage et une augmentation du taux de participation aux USA car les 35-54 ans avaient le plus faible taux de chômage et le plus fort taux de participation de toute la population. A structure constante, le taux de chômage aurait donc du baisser de 0,4\% aux USA consécutivement au vieillissement des baby boomers. Shimer (1998) confirme ces résultats : l'impact direct ainsi calculé de l'évolution démographique expliquerait 0,8 point de la hausse du chômage entre 1954 et 1978 et 0,8 point de la baisse du chômage entre 1978 et 1998.

\section{...sont faibles}

Les effets de composition ainsi calculés sont faibles (de l'ordre de 0,5 point de chômage), mais non négligeables : l'arrivée sur le marché du travail d'une cohorte nombreuse a donc 
tout d'abord un impact positif sur le chômage total, puis un impact négatif. Même si cette méthode ne prend pas en compte l'impact indirect d'une augmentation du nombre des inactifs de plus de 60 ans (retraités) car elle ne considère que l'évolution démographique de la population active, le vieillissement de la population prévu en France doit aussi se caractériser par une baisse de la proportion de 20-30 ans dans la population active. L'effet démographique direct ainsi calculé conduirait donc dans un premier temps en France à une diminution du chômage et une augmentation des taux de participation.

L'application de cette méthode à la France fournit en prospective des résultats d'un ordre de grandeur comparables à ceux obtenus pour les USA : l'évolution démographique contribuerait de manière directe à une diminution de 0,3 point de chômage entre 97 et 2010 . Ce résultat est cependant à relativiser en raison de la proportion croissante de 55-64 ans dans la population ; or le taux d'activité de cette classe d'âge était de $37 \%$ en France en 1997, ce qui est très faible (l'impact sur les taux de participation diffère donc de celui sur les taux de chômage, l'existence de mécanismes de pré-retraites n'étant sans doute pas étranger à ce phénomène). A l'horizon 2030, l'évolution démographique depuis 1997 serait donc neutre par rapport au chômage. La même méthode appliquée rétrospectivement à la France indique que l'évolution démographique directe aurait apporté une diminution du chômage de 0,3 points entre 1979 et 1997 . Cette méthode a donc des limites, en particulier sur longue période : ainsi, le taux de chômage chez les jeunes de moins de 25 ans est passé en France de 13 à 28\% entre 1979 et 1997, tandis que le taux d'activité chutait de 48 à 28\%. Dans un tel contexte, l'impact de l'évolution démographique est insignifiant par rapport à l'évolution des structures du marché du travail, notamment du taux de chômage des différentes tranches d'âge. De même, la baisse constante des taux de participation des plus de 55 ans dans tous les pays industrialisés depuis 40 ans a certainement un impact bien plus fort que la modification de la pyramide des âges.

Pissarides (1989) fournit une autre explication aux différences de chômage entre actifs jeunes et actifs âgés : les voix de ces derniers auraient plus de poids que celles de leurs cadets dans les syndicats. Les âgés accordent dans ce cadre plus de poids au chômage dans leur arbitrage chômage/salaire que les jeunes : en effet, les âgés ont plus à perdre du chômage que les jeunes car ils retrouvent moins facilement du travail et car le passage de l'état de travailleur à l'état de chômeur leur cause un préjudice financier supérieur, en particulier si l'indemnité chômage est forfaitaire comme au Royaume-Uni. Dès lors, le vieillissement de la population se traduirait par une diminution du chômage des deux catégories d'agents car les desiderata des plus âgés l'emportent sur les préférences des jeunes. Ce résultat est certainement à interpréter avec précaution: l'existence de mécanismes du type « first in, last out » combinée à une plus forte syndicalisation des actifs âgés pourrait rendre moins tranché l'impact d'une évolution de la pyramide des âges sur les objectifs des syndicats. 


\subsection{Les liens entre la taille d'une cohorte et son taux de chômage}

Les changements démographiques peuvent aussi avoir des effets indirects sur les taux de chômage selon l'âge. Ainsi, le modèle de croissance néo-classique prédit qu'une augmentation du taux de croissance de la population active réduit le rapport capital sur travail, ce qui accroît les taux d'intérêt et diminue les salaires. Dans un marché frictionnel, les agents réduisent alors leurs efforts de recherche d'emploi : ceci peut résulter en une augmentation du chômage. Shimer (1998) estime que le niveau de ces effets indirects est comparable à celui des effets directs : le Baby Boom expliquerait donc des variations de 2 points de chômage au cours des quarante dernières années. Mais, comme l'auteur le souligne lui-même, les liens ainsi observés entre la taille d'une cohorte et son taux de chômage pourraient en fait traduire un choc macroéconomique exogène (en particulier dans les années 70). Chercher un lien entre la taille d'une cohorte et son taux de chômage de manière absolue pose cependant le problème des comparaisons internationales : des pays de taille très différentes ont des taux de chômage comparables, il semble donc que si une telle relation existe, elle se pose plutôt en termes relatifs. Dans un contexte de marchés segmentés (et donc de rendements décroissants spécifiques aux différentes cohortes), le vieillissement devrait entraîner une amélioration de la situation des jeunes parallèlement à une dégradation de la situation des seniors.

Korenman et Neumark (1997) examinent l'évolution du chômage dans 15 pays et trouvent qu'une augmentation de la taille relative de la cohorte des jeunes actifs augmente le chômage relatif de cette cohorte par rapport aux autres avec une élasticité proche de 0,5. L'utilisation de plusieurs pays et de séries temporelles leur permet d'éliminer les effets d'un choc macroéconomique exogène. Cependant, leur utilisation du taux de chômage relatif empêche de déterminer le mécanisme exact prenant place (.e. une augmentation du chômage des jeunes ou une diminution du chômage des actifs plus âgés). Shimer (1999) résout ce problème en ne considérant que les Etats-Unis, mais en utilisant des données sur les 50 états américains (les chocs entre états étant décorrélés, l'auteur estime aussi régler le problème des chocs exogènes). Il trouve qu'une augmentation de $1 \%$ de la part des jeunes dans la population active entraîne une diminution du chômage des actifs jeunes de $1 \%$ et des actifs plus âgés de $2 \%$. Ces ordres de grandeur sont très élevés. L'impact est en outre renforcé par l'augmentation résultante des taux de participation (élasticités respectives de $1 / 3$ et $1 / 20$ ). L'auteur explique ce résultat par la prise en compte de frictions sur le marché du travail. Une augmentation de la part des jeunes dans la population active augmenterait la fluidité du marché encourageant l'emploi. Shimer explique aussi les différences de résultats entre son étude et celle de Korenman et Neumark par l'existence de mouvements internationaux de capitaux qui annuleraient le mécanisme de l'effet d'encombrement/dilution (i.e. le ratio capital/travail n'évoluerait plus forcément en parallèle avec l'évolution démographique). Les différentes cohortes ne peuvent donc être considérées comme indépendantes : les effets croisés existent et semblent d'un ordre de grandeur comparable aux effets directs. 


\subsection{Vieillissement et rémunération à l'ancienneté}

Lazear (1979) souligne les inégalités de traitement entre jeunes et vieux au niveau de leur rémunération : les agents sont au début de leur carrière payés à un niveau inférieur à leur productivité. Au contraire, les agents plus âgés sont rémunérés à un niveau supérieur à leur productivité. Plusieurs explications ont été données à ce phénomène : la théorie du signal souligne la plus forte incertitude entourant les jeunes actifs quand à leurs réelles performances ; l'existence de rigidités (grilles de salaires, etc.) ou la moindre substituabilité entre agents expérimentés peut expliquer le résultat des actifs âgés ${ }^{14}$. Enfin, les modèles de séniorité (Lazear 1979) expliquent ce phénomène par une volonté d'inciter les jeunes travailleurs à rester dans leur entreprise et à être efficaces, dans l'espoir d'en toucher les dividendes plus tard (l'entreprise respectant ce contrat dans un souci de crédibilité vis-à-vis des générations futures). Il y a donc comme le souligne Lazear (1990) un risque lié au vieillissement: celui d'une forte hausse des salaires, partiellement décorrélée de la progression de la productivité. Cette hausse des salaires réels, ne pouvant être absorbée par une diminution de la rémunération des segments les plus jeunes des actifs, conduirait à une augmentation des pressions inflationnistes ou à une remise en cause du lien entre coût unitaire réel du travail et âge des actifs, ce qui ne serait pas sans conséquences sociales (départs à la retraite, etc.).

A titre d'exemple de l'impact potentiel du maintien inchangé de ces politiques d'ancienneté, Blanchet (1995) montre à l'aide d'un modèle simple (il postule un important effet d'ancienneté pure sur les rémunérations : le rapport salaire sur productivité évoluant de $60 \%$ à $120 \%$ entre 20 et 60 ans) que le taux de marge des entreprises est sensiblement affecté par le vieillissement de la population : il diminuerait d'environ 1/3 d'ici 2040. Pour maintenir leurs marges, les entreprises devraient revoir leurs politiques de rémunérations. $\mathrm{Si}$ les entreprises réagissaient à cette évolution en remettant en cause ce lien, on peut s'attendre à une forte réévaluation des salaires des actifs jeunes (vers leur niveau de productivité) et une diminution des salaires des actifs âgés : le vieillissement est donc susceptible de remettre en cause les politiques de rémunération à l'ancienneté.

Au niveau empirique, l'impact positif de l'âge sur les salaires n'est cependant pas accepté par tous. Ainsi, les études de l'OCDE (1998) indiquent que le salaire maximum serait atteint par les actifs entre 50 et 55 ans, payés $40 \%$ de plus que les actifs débutants et $10 \%$ de plus que les actifs de plus de 55 ans. Cette prime à l'ancienneté se serait accrue depuis vingt ans. Mais Lhéritier (1992) trouve, pour les pays de l'OCDE, un effet positif de l'âge sur les salaires, croissant avec les niveaux de qualification, jusqu'à 50 ans, puis négatif au delà de 50 ans. Ce résultat négatif pourrait cependant s'expliquer par un biais de sélection car l'auteur ne prend pas en compte les promotions des agents : ses techniciens de 50 ans sont donc ceux qui n'ont pas été promus cadre durant leur carrière. Lollivier et Payen (1990) éliminent ce problème par un suivi des agents : l'impact ainsi obtenu de l'âge sur les salaires est alors toujours positif jusqu'à la retraite. De même, Crepon et al. (2002)

\footnotetext{
14 La théorie du capital humain explique la relation croissante entre salaires et âge par les effets d'apprentissage ; il faudrait supposer que la productivité est imparfaitement observée pour que cette théorie s'applique ici.
} 
confirment l'existence de ces « primes à l'ancienneté » pour la France. Il est à noter que de fortes spécificités nationales existent : ainsi, au Japon, la décrue des salaires des actifs de plus de 50 ans serait en bonne partie due à leur changement de situation. Passé les 50 ans, de nombreux salariés quitteraient en effet la grande entreprise où ils étaient employés pour travailler dans de plus petites entités moins productives pour une rémunération moindre.

Les travailleurs qualifiés apparaissent moins substituables selon leur âge que les travailleurs non qualifiés (Welch 1979), car l'emploi qualifié accorderait un poids plus important aux effets d'expérience. Cependant, le développement du travail qualifié ne saurait expliquer à lui seul le développement des effets d'ancienneté, ceux-ci s'étant accrus pour toutes les catégories.

Comme vu précédemment, une autre explication aux différences de salaires entre actifs jeunes et actifs âgés est donnée par Pissarides (1989). Ces arguments de type insiders/outsiders permettent d'expliquer que le salaire des actifs âgés soit supérieur à celui des actifs jeunes quelles que soient les productivités ou les tailles relatives des différentes cohortes. Dans ce cadre, le vieillissement de la population se traduirait par une diminution du salaire des deux catégories d'agents. Les précautions soulignées sur le chômage restent cependant de mise.

\subsection{Salaires et taille d'une cohorte}

En plus de cet effet de structure, il existe un effet de la taille absolue de la cohorte sur les salaires. Avec une fonction de production à rendements constants, une augmentation de la taille d'une cohorte entraîne mécaniquement une diminution du salaire de cette cohorte. Welch (1979) confirme empiriquement ce résultat pour les Etats-Unis : une augmentation de $10 \%$ de la taille d'une cohorte se traduirait par une diminution des salaires des membres de cette cohorte : de $9 \%$ pour les « college graduate » et de $4 \%$ pour les personnes ayant fait des études supérieures. Ces résultats sont corroborés par l'étude de Murphy et al. (1988) qui montre, de plus, que ces effets diminuent avec l'expérience : les actifs âgés seraient donc moins sensibles aux effets induits par la taille de leur cohorte que les actifs plus jeunes. Ceci pourrait toutefois s'expliquer par les spécificités du chômage des actifs âgés (voir supra) ou par le fait que les travailleurs âgés sont moins substituables entre eux que les travailleurs plus jeunes car leur expérience professionnelle les individualiserait plus. Ces effets existent donc et ne sont pas négligeables, même si ils sont inférieurs aux différentiels de salaires induits par le secteur d'occupation ou le sexe.

En plus de cette corrélation entre le niveau de salaires et la taille absolue des cohortes, il semble que les salaires relatifs des différents agents soient reliés à la taille relative de la cohorte à laquelle ils appartiennent. Katz et Murphy (1992) trouvent ainsi une élasticité de 2,9 entre la taille relative d'une cohorte et le salaire relatif de cette cohorte. Ce résultat est confirmé par Macunovich (1998) pour qui l'évolution de la pyramide des âges aux EtatsUnis suffirait à expliquer l'évolution de la prime d'expérience ainsi qu'une forte part de l'évolution de la prime à la qualification. Ces effets induits par la taille relative d'une cohorte sur le salaire relatif de cette cohorte se retrouvent au niveau international. Higgins et Williamson (1999) montrent que les différentes structures par âge des populations expliquent une part des différences dans les coefficients d'inégalité entre les pays. Là 
encore, les effets de la taille relative de la cohorte sur les salaires relatifs semblent diminuer avec l'âge. Ces résultats ne permettent cependant pas de trancher définitivement sur le degré de segmentation du marché du travail.

En termes prospectifs, les sociétés vieillissantes font face à un écueil : pour des raisons d'équilibre budgétaire des systèmes de retraite, il faudrait repousser l'âge de la retraite afin d'allonger les durées de cotisation ; en cas de persistance des politiques de rémunérations à l'ancienneté, les entreprises seraient incitées à se séparer le plus vite possible de leurs salariés âgés ; mais l'abandon de telles politiques de rémunération augmenterait les incitations à partir de plus en plus tôt pour les salariés et se heurterait à de vives résistances.

\subsection{Mobilité, qualification et productivité : quels liens avec l'âge ?}

La mobilité des agents permet d'atténuer l'impact d'un choc spécifique (touchant une région ou un secteur particulier). Tant sur le plan géographique que sur le plan occupationnel, la mobilité des jeunes actifs est supérieure à celle des travailleurs plus âgés. Plusieurs raisons expliquent ce phénomène: la mobilité géographique est corrélée négativement avec la possession de biens immobiliers ; or les jeunes sont moins souvent propriétaires que leurs aînés. Ils sont de plus moins souvent chargés de famille. De même, leurs rentes de situation sont souvent faibles (manque d'expérience); ils hésitent donc moins à changer de secteur d'occupation que les agents plus âgés qui perdraient ce faisant une partie du bénéfice apporté par leur expérience (mobilité horizontale).

Ces résultats sont confirmés par Shimer (1999) qui trouve qu'une augmentation de $1 \%$ de la part des jeunes dans la population active entraîne un accroissement de $0,8 \%$ des créations d'emploi et de $0,7 \%$ des destructions d'emploi. Un marché du travail plus jeune serait donc plus fluide. Il faut noter que ce résultat (et la théorie sous-jacente) restent parfaitement valables en cas de non segmentation du marché du travail (i.e. jeunes et vieux parfaitement substituables pour les entreprises) : il suffit de considérer qu'il faut du temps pour trouver un bon appariement entre les caractéristiques d'un salarié et son emploi (Pissarides, 1989). Les marchés du travail plus âgés sont donc alors plus rigides, ce qui est néfaste pour l'emploi.

La mobilité n'est pas seulement géographique ou horizontale : elle est aussi verticale (promotions, accession à des postes de responsabilité). Or comme le soulignent Bös et Weizsäcker (1989), le risque existe dans une société vieillissante de voir un ralentissement de cette mobilité verticale, avec surpopulation des échelons supérieurs (les promotions automatiques à l'ancienneté posent d'ailleurs déjà des problèmes dans plusieurs secteurs en France). De nombreux jeunes pourraient donc quitter leur travail ou faire moins d'efforts de carrière en raison d'un manque de débouchés. De ce point de vue, le vieillissement de la population aura donc des conséquences néfastes sur l'économie : le chômage serait plus élevé de par la moindre adéquation entre offre et demande de travail et les chocs seront plus difficiles à absorber. L'argument d'encombrement des postes de responsabilité pourrait aussi avoir un effet négatif sur les qualifications: dès lors que celles-ci résultent d'investissements en capital humain, les incitations à se qualifier diminueraient. Il faut toutefois noter que cet effet semble bien faible au regard de l'augmentation constatée depuis 15 ans des primes à la qualification. De plus, un tel phénomène d'engorgement des 
Evolutions démographiques et marchés du travail : des liens complexes parfois contradictoires

postes de responsabilité pourrait inciter de nombreux actifs jeunes à devenir entrepreneurs, ce qui aurait des conséquences positives pour l'emploi et la productivité.

Les générations successives sont de plus en plus qualifiées : les durées de scolarisation ne cessent d'augmenter. Pourtant, la relation entre productivité et âge est ambiguë : aux effets d'apprentissage s'oppose une adéquation supérieure de la main d'œuvre jeune aux métiers nouvellement créés. Bös et Weizsäcker (1989) soulignent ainsi que l'impact négatif du vieillissement sur la productivité croît avec la vitesse d'introduction de nouvelles technologies. En cas de progrès technique rapide et soutenu, une population âgée constituerait donc un handicap, de par son manque d'adaptabilité et l'inadéquation de son expérience. Parmi les autres arguments avancés à la diminution de productivité des salariés les plus âgés, on peut aussi citer la dépréciation des compétences et des qualifications. Blanchet et Kessler (1990) confirment ce profil en cloche de la relation à un niveau individuel entre structure par âge et productivité. Ce type de relation est cependant difficile à prouver car pour les raisons précitées, les salaires ne peuvent être utilisés pour approximer les niveaux de productivité.

Le lien entre qualification et âge se complique de par l'existence d'un apprentissage continu : l'existence de standards de compétences impose à tout salarié quelque soit son âge de les maîtriser. La dépréciation des compétences des salariés les plus âgés est donc fortement liée à l'inexistence de formations professionnelles tout au long de leur carrière. En l'absence de telles formations, la logique de rendements croissants sur les qualifications profiterait aux jeunes actifs et dégraderait la position des plus âgés. A un niveau plus général, les économies actuelles semblent de plus en plus fondées sur le capital humain et la connaissance : ceci laisse entrevoir selon l'OCDE une accentuation du problème de « l'employabilité » des personnes âgées dans la tranche des non-qualifiés ; par contre, dans les métiers basés sur le savoir, le vieillissement ne serait pas un handicap.

De manière plus générale, les liens entre croissance économique de plus ou moins long terme et évolution démographique ont fait l'objet de nombreuses études ${ }^{15}$. Plusieurs mécanismes parmi lesquels les effets de dilution du capital, l'adaptation des technologies aux contraintes démographiques ou encore la présence d'effets d'échelle ont été identifiés. Cependant, cette multiplicité dans les mécanismes théoriques ne transparaît guère dans les évolutions des pays industrialisés.

\section{CONCLUSION}

Les impacts de l'évolution démographique se décomposent en impacts directs sur le marché du travail (via l'offre et la demande) et impacts indirects via le marché des biens, l'épargne, les inégalités, la croissance de long terme, etc. Quoique potentiellement très important au niveau théorique, l'ordre de grandeur de ces impacts indirects est cependant généralement faible dans les faits par rapport aux impacts directs. L'effet du ralentissement de la croissance de la population en âge de travailler sur le chômage de moyen terme dépend de la rigidité des salaires et des prix, des mécanismes de fixation des salaires, et donc du type

${ }^{15}$ Voir Jones (1999) ou Blanchet (1991) pour des exposés de ces différents éléments. 
de chômage existant dans l'économie. Mais, loin de la sagesse commune, il ne faut pas attendre de miracle de l'évolution démographique en volume : la baisse résultante de l'offre de travail ne saurait être, surtout à terme, la solution au problème du chômage de masse qui a touché les économies européennes.

Dans le même temps, le vieillissement de la population en âge de travailler entraînera à structures de marchés constantes une modification des situations relatives des différentes cohortes sur le marché du travail. La mesure de ces effets dépend fortement des segmentations existant sur le marché du travail (en fonction de l'âge et de la qualification). L'impact de ce vieillissement sera plus fort si l'on ne considère pas les évolutions de fond qui l'accompagnent. Or la comparaison des simples ordres de grandeur montre que ces évolutions doivent être prises en compte. Il y a plusieurs exemples de tels mécanismes de réactions endogènes au premier rang desquels une modification des systèmes de retraite dans de nombreux pays dans un souci d'équilibre des régimes, ou encore une modification des politiques de rémunération à l'ancienneté actuellement en vigueur. Ces mécanismes d'auto-ajustement sont suffisamment forts pour pouvoir le cas échéant neutraliser une bonne part des impacts dus au vieillissement. L'identification des rigidités empêchant ces mécanismes de se réaliser devrait donc être une partie intégrante de toute étude visant à quantifier l'impact de l'évolution démographique car c'est en présence de telles rigidités que cet impact sera le plus important.

L'évolution démographique à venir aura des conséquences importantes sur le fonctionnement des marchés du travail des économies industrialisées, et ce, même si cet impact ne transparaît que peu dans les chiffres : ce sont souvent les règles ou le contrat social en vigueur qui évolueront. 
Evolutions démographiques et marchés du travail : des liens complexes parfois contradictoires

\section{RET E R E N CES}

Aglietta M., Blanchet D. et Héran F. (2002), "Démographie et Economie", la documentation française editeur, Vol 35 du rapport au Premier Ministre, Paris, Conseil d'Analyse Economique.

Blanchard O. et Summers L. (1986), "Hysteresis and the European unemployment problem”, NBER Macroeconomics Annual, vol. 1, Cambridge, MIT Press, p. 15-77.

Blanchet D. (1991), “Modélisation démo-économique”, Travaux et Documents INED, Cahier $\mathrm{n}^{\circ} 130$,.

Blanchet D. (1995), "Le vieillissement de la population active doit-il affecter les politiques de formation et les politiques salariales ?", dans Cochemé et Legros, Les retraites, genèse, acteurs, enjeux, Armand Colin.

Blanchet D. (2001), "L'impact des changements démographiques sur la croissance et le marché du travail : faits, théories et incertitudes", mimeo, INSEE.

Blanchet D. et Kessler D. (1990), "Prévoir les effets économiques du vieillissement", Economie et Statistique, $n^{\circ} 233$.

Bodier M. (1999), "Les effets d'âge et de génération sur le niveau et la structure de la consommation”, Economie et Statistique, n³24-325.

Bös D. and von Weizsäcker R.K. (1989), "Economic consequences of an aging population”, European Economic Review, vol 33.

Brown C., Gilroy C. and Kohen A. (1982), "The effect of the minimum wage on employment and unemployment", Journal of Economic Litterature, vol 20, (2).

Cahuc P. et Zylberberg A. (1999), "Le modele WS-PS", Annales d'Economie et de Statistique, 0(53).

Château J. (1999), “Le vieillissement démographique, l’épargne et la croissance : approche macroéconomique", Note ronéotypée du Commissariat Général au Plan, Service Economique, Financier et International.

Château J., Guérin J.L. et Legros F. (2002), "Demographic evolutions and unemployment : a french labour market analysis with generations of workers", mimeo CEPII, 2002.

Clark R.L. and Spengler J.J.(1980), "Economics of individual and population aging", Cambridge University Press. 
COR (2001), "Retraites: Renouveler le contrat social entre les générations: Orientations et débats", la documentation française editeur, Vol 1 du rapport au Premier Ministre, Paris, Conseil d'Orientation des Retraites.

Crepon B., Deniau N. et Perez-Duarte S. (2002), "Wages, productivitry and workers characteristics: a French perspective", mimeo CREST INSEE.

Cutler D., Poterba J., Sheiner L. and Summers L.H. (1990), “An aging society : opportunity or challenge ?", Brookings Papers on Economic Activity, vol 1.

Darmon D., Hourriez J.M. et L'Hardy P. (1991), “Consommation : l'effet du vieillissement”, Economie et Statistique, $\mathrm{n}^{\circ} 243$.

Diamond P.A. and Hausman J.A. (1984), "The retirement and unemployment behaviour of elder men", in Aaron H.J. and Burtless G. eds Retirement and economic behaviour, Brookings Institute.

Dolado J., Kramarz F., Machin S., Manning A., Margolis D. and Teulings C. (1996), "The economic impact of minimum wages in Europe", Economic Policy-An European Forum, 0 (23).

Domingues Dos Santos M. (2001), "Vieillissement démographique et chômage", Revue française d'économie, 16, (1), p. 3-50.

Flaim P. (1990), "Population changes, the Baby Boom and the unemployment rate", Monthly Labor Review.

Fuchs V. (1998), "Provide, Provide : The economics of aging”, NBER Working Paper 6642 , July.

Higgins M. and Williamson J.G. (1999), "Explaining inequality the world round : cohort size, kuznets curves, and openness", NBER Working Paper 7224.

Hurd M. (1991), "The effects of demographic trends on consumption, saving and government expenditures in the U.S.", NBER Working Paper 4601.

Jones C.I. (1999), "Growth : with or without scale effects ?", American Economic Review, vol 89, (2).

Katz L. and Murphy K. (1992), “Changes in relative wages, 1963-1987 : supply and demand factors", Quarterly Journal of Economics, vol 107.

Kidd D. and Oswald A. (1987), “A dynamic model of trade union behaviour”, Economica, vol 54, (215), p. 355-65. 
Evolutions démographiques et marchés du travail : des liens complexes parfois contradictoires

Kohl R. and O’Brien P. (1998), “The macroeconomics of ageing, pensions and savings : a survey”, OECD Working Paper 200.

Lazear E. (1979), "Why is there mandatory requirement", Journal of Political Economy, vol 87.

Lazear E. (1990), "Adjusting to an aging labor force", in D.Wise ed. Issues in the Economics of aging, University of Chicago Press.

Lhéritier (1992), “Les déterminants du salaire”, Economie et Statistique, vol 257.

Linbeck A. et Snower D. (1987), "Union activity, unemployment persistence, and wageemployment ratchets", European Economic Review, vol 31, February.

Loisy C. (1999), “Taux d’épargne par catégorie de ménages”, Données Sociales, chapitre 6, p. 294-300.

Lollivier S. et Payen J.F. (1990) "Hétérogénéïté des carrières individuelles mesurées sur données de panel”, Economie et Prévision, vol 92-93.

Macunovich D.J. (1998), " Relative cohort size and inequality in the United States", American Economic Review, vol 88, 1998.

Marchand O. et Salzberg L. (1996), "La gestion des âges à la française, un handicap pour l'avenir ?", Données Sociales.

Masson P.R., Bayoumi T. and Samiei H. (1996), "International evidence on the determinants of private saving", CEPR Discussion Paper 1368.

Murphy K., Plant M. and Welch F. (1988), "Cohort size and earnings in the United States" in R.Lee, W.Arthur and G.Rodgers eds. Economics of changing age distributions in developped countries, Clarendon Press Oxford.

Neumark D. and Wascher W. (1999), “A cross-national analysis of the effects of minimum wages on youth employment”, NBER Working Paper 7299.

OCDE (1998), Perspectives de l'emploi.

Phelps E. (1994), "Structural slumps : the modern equilibrium theory of unemployment, interest, and assets", in collaboration with Hian Teck Hoon, Kanaginis and Zoega, Harvard Univesity Press.

Phelps E. and Zoega G. (1998), "Natural rate theory and OECD unemployment", Economic Journal, vol 108, (448), p. 782-801. 
Pissarides C.A. (1989), "Unemployment consequences of an aging population”, European Economic Review, vol 33.

Quintreau B. (2001), “Age et emploi à l'horizon 2010”, Journal Officiel, vol 20 du rapport, Paris, Conseil Economique et Social.

Sanwa Bank Limited (1994), "Demographic aging in Japan. Influence on consumption and Markets”, Economic Letter.

Shimer R. (1998), "Why is the U.S. unemployment rate so much lower?", NBER Macroeconomics Annual, vol 13.

Shimer R. (1999), "The impact of young workers on the aggregate labor market", NBER Working Paper 7306.

Tyrvainen T. (1995), "Real wage resistance and unemployment : multivariate analysis of cointegrating relations in 10 OECD countries", OECD Working Paper, $n^{\circ} 135$.

Weil D. (1994), "The saving of the elderly in micro and macro data", Quarterly Journal of Economics, vol, February 1994.

Welch F. (1979), "Effects of cohort size on earnings : the Baby Boom babies financial bust", Journal of Political Economy, vol 87. 


\section{LISTE DES DOCUMENTS DE TRAVAIL REALISES PAR LE CEPII ${ }^{16}$}

2002-15 Exchange rate Regimes and Sustainable Parities for

V. Coudert \& CEECs in the Run-up to EMU Membersuip

C. Couharde

2002-14 When are Structural Deficits Good Policies?

J. Chateau

2002-13 Projections démographiques de quelques pays de

R. Sleiman l'Union Européenne (Allemagne, France, Italie, Royaume-Uni, Pays-Bas, Suède)

2002-12 Regional Trade Integration in Southern Africa

S. Chauvin \&

G. Gaulier

2002-11 Demographic Evolutions and Unemployment: an Analysis of French Labour Market with Workers Generations

2002-10 Liquidité et passage de la valeur

P. Villa

2002-09 Le concept de coût d'usage Putty-Clay des biens durables

M.G. Foggea \& P. Villa

2002-08 Mondialisation et régionalisation : le cas des industries du textile et de l'habillement

M. Fouquin, P. Morand R. Avisse G. Minvielle \& P. Dumont

2002-07 The Survival of Intermediate Exchange Rate Regimes

A. Bénassy-Quéré \&

B. Coeuré

2002-06 Pensions and Savings in a Monetary Union: An A. Jousten \& F. Legros Analysis of Capital Flow

2002-05 Brazil and Mexico's Manufacturing Performance in International Perspective, 1970-1999

N. Mulder, S. Montout \& L. Peres Lopes

2002-04 The Impact of Central Bank Intervention on Exchange-Rate Forecast Heterogeneity

M. Beine,

A. Benassy-Quéré,

E. Dauchy \&

R. MacDonald

\footnotetext{
${ }^{16}$ Working papers are circulated free of charge as far as stocks are available; thank you to send your request to CEPII, Sylvie Hurion, 9, rue Georges-Pitard, 75015 Paris, or by fax : (33) 0153685504 or by e-mail Hurion@cepii.fr. Also available on: Nwww.cepii.fr. Working papers with* are out of print. They can nevertheless be consulted and downloaded from this website. 16

Les documents de travail sont diffusés gratuitement sur demande dans la mesure des stocks disponibles. Merci d'adresser votre demande au CEPII, Sylvie Hurion, 9, rue Georges-Pitard, 75015 Paris, ou par fax : (33) 0153685504 ou par e-mail Hurion@cepii.fr. Egalement disponibles sur : Ilwww.cepii.fr. Les documents de travail comportant * sont épuisés. Ils sont toutefois consultable sur le web CEPII.
} 
2002-04 The Impact of Central Bank Intervention on Forecast Heterogeneity

2002-03 Impacts économiques et sociaux de l'élargissement pour l'Union européenne et la France

2002-02 China in the International Segmentation of Production Processes

2002-01 Illusory Border Effects: Distance Mismeasurement Inflates Estimates of Home Bias in Trade

2001-22 Programme de travail du CEPII pour 2002

2001-21 Croissance économique mondiale: un scénario de référence à l'horizon 2030

2001-20 The Fiscal Stabilization Policy under EMU - An Empirical Assessment

2001-19 Direct Foreign Investments and Productivity Growth in Hungarian Firms, 1992-1999

2001-18 Market Access Maps: A Bilateral and Disaggregated Measure of Market Access

2001-17 Macroeconomic Consequences of Pension Reforms in Europe: An Investigation with the INGENUE World Model

2001-16* La productivité des industries méditerranéennes

2001-15 Marmotte: A Multinational Model

2001-14 The French-German Productivity Comparison Revisited: Ten Years After the German Unification

2001-13* The Nature of Specialization Matters for Growth: An Empirical Investigation
M. Beine,

A. Benassy-Quéré,

E. Dauchi \&

R. MacDonald

M.H. Bchir \&

M. Maurel

F. Lemoine \&

D. Ünal-Kesenci

K Head \& T. Mayer

N. Kousnetzoff

A. Kadareja

J. Sgard

A. Bouët, L. Fontagné, M. Mimouni \& X. Pichot

Equipe Ingénue

A. Chevallier \& D. Ünal-Kesenci

L. Cadiou, S. Dees,

S. Guichard,

A. Kadareja,

J.P. Laffargue \&

B. Rzepkowski

L. Nayman \&

D. Ünal-Kesenci

I. Bensidoun, G. Gaulier

\& D. Ünal-Kesenci 
2001-12 Forum Economique Franco-Allemand - DeutschFranzösisches Wirtschaftspolitisches Forum, Political Economy of the Nice Treaty: Rebalancing the EU Council and the Future of European Agricultural Policies, $9^{\text {th }}$ meeting, Paris, June $26^{\text {th }} 2001$

2001-11 Sector Sensitivity to Exchange Rate Fluctuations

2001-10* A First Assessment of Environment-Related Trade Barriers

2001-09 International Trade and Rend Sharing in Developed and Developing Countries

2001-08 Economie de la transition : le dossier

2001-07 Exit Options for Argentina with a Special Focus on Their Impact on External Trade

2001-06 Effet frontière, intégration économique et 'Forteresse Europe'

2001-05 Forum Économique Franco-Allemand - DeutschFranzösisches Wirtschaftspolitisches Forum, The Impact of Eastern Enlargement on EU-Labour Markets and Pensions Reforms between Economic and Political Problems, $8^{\text {th }}$ meeting, Paris, January 16 2001

2001-04 Discrimination commerciale : une mesure à partir des flux bilatéraux

2001-03* Heterogeneous Expectations, Currency Options and the Euro/Dollar Exchange Rate

2001-02 Defining Consumption Behavior in a Multi-Country Model

2001-01 Pouvoir prédictif de la volatilité implicite dans le prix des options de change

2000-22 Forum Economique Franco-Allemand - DeutschFranzösisches Wirtschaftspolitisches Forum, Trade Rules and Global Governance: A long Term Agenda and The Future of Banking in Europe, $7^{\text {th }}$ meeting, Paris, July 3-4 2000
M. Fouquin, K. Sekkat,

J. Malek Mansour,

N. Mulder \&

L. Nayman

L. Fontagné, F. von

Kirchbach \&

M.Mimouni

L. Fontagné \&

D. Mirza

G. Wild

S. Chauvin

T. Mayer

G. Gaulier

B. Rzepkowski

O. Allais, L. Cadiou \& S. Dées

B. Rzepkowski 
2000-21 The Wage Curve: the Lessons of an Estimation Over a Panel of Countries

2000-20 A Computational General Equilibrium Model with Vintage Capital

2000-19 Consumption Habit and Equity Premium in the G7 Countries

2000-18 Capital Stock and Productivity in French Transport: An International Comparison

2000-17 Programme de travail 2001

2000-16 La gestion des crises de liquidité internationale : logique de faillite, prêteur en dernier ressort et conditionnalité

2000-15 La mesure des protections commerciales nationales

2000-14 The Convergence of Automobile Prices in the European Union: An Empirical Analysis for the Period 1993-1999

2000-13* International Trade and Firms' Heterogeneity Under Monopolistic Competition

2000-12 Syndrome, miracle, modèle polder et autres spécificités néerlandaises : quels enseignements pour l'emploi en France?

2000-11 FDI and the Opening Up of China's Economy

2000-10 Big and Small Currencies: The Regional Connection

2000-09* Structural Changes in Asia And Growth Prospects After the Crisis

2000-08 The International Monetary Fund and the International Financial Architecture

2000-07 The Effect of International Trade on Labour-Demand Elasticities: Intersectoral Matters

2000-06 Foreign Direct Investment and the Prospects for Tax Co-Ordination in Europe

2000-05 Forum Economique Franco-Allemand - DeutschFranzösisches Wirtschaftspolitisches Forum, Economic Growth in Europe Entering a New Area?/The First Year of EMU, $6^{\text {th }}$ meeting, Bonn,
S. Guichard \&

J.P. Laffargue

L. Cadiou, S. Dées \& J.P. Laffargue

O. Allais, L. Cadiou \& S. Dées

B. Chane Kune \& N. Mulder

J. Sgard

A. Bouët

G. Gaulier \& S. Haller

S. Jean

S. Jean

F. Lemoine

A. Bénassy-Quéré \&

B. Coeuré

J.C. Berthélemy \& S. Chauvin

M. Aglietta

S. Jean

A. Bénéssy-Quéré, L. Fontagné \& A. Lahrèche-Révil 
Evolutions démographiques et marchés du travail : des liens complexes parfois contradictoires

January 17-18, 2000

2000-04* The Expectations of Hong Kong Dollar Devaluation and their Determinants

B. Rzepkowski

2000-03 What Drove Relative Wages in France? Structural Decomposition Analysis in a General

Equilibrium Framework, 1970-1992

2000-02 Le passage des retraites de la répartition à la capitalisation obligatoire : des simulations à l'aide d'une maquette

2000-01* Rapport d'activité 1999

1999-16 Exchange Rate Strategies in the Competition for Attracting FDI

A. Bénassy-Quéré,

L. Fontagné \&

A. Lahrèche-Révil

D. Pianelli \&

1999-15 Groupe d'échanges et de réflexion sur la Caspienne. Recueil des comptes-rendus de réunion (déc. 97- oct. 98)"

1999-14 The Impact of Foreign Exchange Interventions: New Evidence from FIGARCH Estimations

G. Sokoloff

M. Beine,

A. Bénassy-Quéré \&

C. Lecourt

1999-13 Forum Economique Franco-Allemand - DeutschFranzösisches Wirtschaftspolitisches Forum, Reduction of Working Time/Eastward Enlargment of the European Union, $5^{\text {th }}$ meeting, Paris, July 6-7 1999

1999-12* A Lender of Last Resort for Europe

M. Aglietta

1999-11* La diversité des marchés du travail en Europe: Quelles conséquences pour l'Union Monétaire; Deuxième partie: Les implications macroéconomiques de la diversité des marchés du travail

1999-10* La diversité des marchés du travail en Europe: Quelles conséquences pour l'Union Monétaire; Première partie : La diversité des marchés du travail dans les pays de l'Union Européenne

1999-09 The Role of External Variables in the Chinese Economy; Simulations from a macroeconometric model of China

1999-08 Haute technologie et échelles de qualité : de fortes asymétries en Europe

L. Cadiou, S. Guichard \& M. Maurel

L. Cadiou \&

S. Guichard

S. Dees

L. Fontagné, M. Freudenberg \& D. Ünal-Kesenci 
1999-07 The Role of Capital Accumultion, Adjustment and Structural Change for Economic Take-Off: Empirical Evidence from African Growth Episodes

1999-06 Enterprise Adjustment and the Role of Bank Credit in Russia: Evidence from a 420 Firm's Qualitative Survey

1999-05 Central and Eastern European Countries in the International Division of Labour in Europe

1999-04 Forum Economique Franco-Allemand - Economic Policy Coordination $-4^{\text {th }}$ meeting, Bonn, January 11121999

1999-03 Models of Exchange Rate Expectations: Heterogeneous Evidence From Panel Data

1999-02 Forum Economique Franco-Allemand - Labour Market \& Tax Policy in the EMU

1999-01 Programme de travail 1999
J.C. Berthélemy \&

L. Söderling

S. Brana, M. Maurel \& J. Sgard

M. Freudenberg \& F. Lemoine

A. Bénassy-Quéré, S. Larribeau \& R. MacDonald 


\section{DOCUMENTS DE TRAVAIL / WORKING PAPERS}

Si vous souhaitez recevoir des Documents de travail, merci de remplir le coupon-réponse ci-joint et de le retourner à :

Should you wish to receive copies of the CEPII's Working papers, just fill the reply card and return it to:

\section{Sylvie HURION:}

CEPII - 9, rue Georges-Pitard - 75015 Paris - Fax : 01.53.68.55.04

M./Mme / Mr./Mrs

Nom-Prénom / Name-First name

Titre / Title

Service / Department

Organisme / Organisation

Adresse / Address

Ville \& CP / City \& post code

Pays / Country. Tél.

Désire recevoir les Document de travail du CEPII $n^{\circ}$ - Wish to receive the CEPII's Working Papers No:. 